(n)

\title{
The compatibility of additional symmetry and gauge transformations for the constrained discrete Kadomtsev-Petviashvili hierarchy
}

Maohua Li, Jipeng Cheng, Jingsong He

To cite this article: Maohua Li, Jipeng Cheng, Jingsong He (2015) The compatibility of additional symmetry and gauge transformations for the constrained discrete Kadomtsev-Petviashvili hierarchy, Journal of Nonlinear Mathematical Physics 22:1, 1731, DOI: https://doi.org/10.1080/14029251.2015.996436

To link to this article: https://doi.org/10.1080/14029251.2015.996436

Published online: 04 January 2021 


\title{
The compatibility of additional symmetry and gauge transformations for the constrained discrete Kadomtsev-Petviashvili hierarchy
}

\author{
Maohua Li \\ Department of Mathematics, Ningbo University, \\ Ningbo, 315211 Zhejiang, China \\ Institute for Theoretical Physics, KU Leuven, \\ 3001 Leuven, Belgium \\ limaohua@nbu.edu.cn \\ Jipeng Cheng \\ Department of Mathematics, China University of Mining and Technology, \\ Xuzhou, 221116 Jiangsu, China \\ chengjp@cumt.edu.cn \\ Jingsong $\mathrm{He}^{*}$ \\ Department of Mathematics, Ningbo University, \\ Ningbo, 315211 Zhejiang, China \\ hejingsong@nbu.edu.cn
}

Received 28 July 2014

Accepted 14 September 2014

\begin{abstract}
In this paper, the compatibility between the gauge transformations and the additional symmetry of the constrained discrete Kadomtsev-Petviashvili hierarchy is given, which preserves the form of the additional symmetry of the cdKP hierarchy, up to shifting of the corresponding additional flows by ordinary time flows.
\end{abstract}

Keywords: constrained discrete KP hierarchy; gauge transformation; additional symmetry.

2000 Mathematics Subject Classification: 35Q53, 37K10, 37K40

\section{Introduction}

The discrete Kadomtsev-Petviashvili (dKP) hierarchy ${ }^{a}$ [15, 17, 23, 25, 29] is an attractive research object in the field of the discrete integrable systems. The dKP hierarchy is defined by means of the difference derivative $\Delta$ instead of the usual derivative $\partial$ with respect of $x$ in a classical system $[10,11]$, and the continuous spatial variable is replaced by a discrete variable $n$. By using a nonuniform shift of space variable, the $\tau$-function of KP hierarchy implies a special kind of $\tau$-function for the dKP hierarchy [17]. With the symmetry constraint or symmetry reduction technique, which was used in the continuous KP hierarchy $[6,7,24]$, the constrained discrete KP (cdKP) hierarchy is truncated dKP hierarchy by adding a constrained operator form (see (2.14)) on the Lax operator $L$ of the dKP hierarchy [27]. And the discrete nonlinear Schrödinger equation and other equations can be derived from it.

\footnotetext{
*Corresponding author

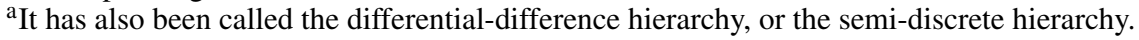


There are many methods for constructing the solutions of the integrable systems for the continuous KP hierarchy $[3,4,19,31,33,34,37,43,45]$. Among these methods, the gauge transformation is one of the important methods to construct the solutions of the integrable systems for the continuous KP hierarchy [3, 4, 19,33,34,37], the dKP hierarchy [27, 30,35] and the cdKP hierarchy [27], which in fact reflects the intrinsic integrability of the KP hierarchy and dKP hierarchy. Chau et al [3] introduce two kinds of elementary gauge transformation operators: the differential type $T_{D}$ and the integral type $T_{I}$. By now, the gauge transformations of many integrable hierarchies related to KP hierarchy have been derived, for example, the constrained KP (cKP) hierarchy $[4,6,7,20,24,34,44]$, the constrained BKP and CKP hierarchy [21, 33] (cBKP and cCKP), the dKP hierarchy [30,35], the cdKP hierarchy [27], the $q$-KP hierarchy $[18,42]$ and so on. The additional symmetry $[1,5,8,12,14,16,22,26,36,38-41]$ is a kind of symmetry depending explicitly on the space and time variables, involved in so-called string equation and the generalized Virasoro constraints in matrix models of the $2 \mathrm{~d}$ quantum gravity (see $[11,32]$ and references therein). Regarding the possible application of the additional symmetry flows of the KP hierarchy in physics, it is natural to ask whether these flows are compatible with the gauge transformation. It is a highly non-trivial question because the gauge transformation is only defined to be consistent with ordinary KP flows. For example, Ref. [2] has shown the compatibility between the the differential type of gauge transformation and the additional symmetry flow of cKP hierarchy separately, up to a shift of ordinary flow of cKP hierarchy. In order to construct the additional symmetry flows of the cKP hierarchy from the corresponding flows of the KP hierarchy, it is necessary to do a remarkable amendment [2] in its definition. So it is an interesting problem to show the compatibility between the gauge transformations and the additional symmetry of the cdKP hierarchy. The additional symmetry flows for the cdKP hierarchy are constructed in $[9,28]$ through a subtle modification of the standard additional symmetry flows by adding a complicated term, which form a Virasoro type algebraic structure [28]. And the action of the Virasoro symmetry on the tau function of the cdKP hierarchy is also derived [9].

In this paper, it is shown that the additional symmetry flows for the cdKP hierarchy commute with the integral type and difference type gauge transformations preserving the form of the additional symmetry of the cdKP hierarchy, up to shifting of the corresponding additional flows by ordinary time flows, which reflects the compatibility between the two types of the gauge transformations and the additional symmetries of the cdKP hierarchy.

This paper is organized as follows. Some backgrounds on the dKP hierarchy are given in Section 2. Then the two types gauge transformation operators of the cdKP hierarchy are reviewed in Section 3. And the additional symmetry for the cdKP hierarchy are reviewed in Section 4. In Section 5, it is derived that the additional symmetry commute with the gauge transformations preserving the form of the additional symmetry of the cdKP hierarchy.

\section{Background On The dKP Hierarchy}

Some basic facts about the dKP hierarchy are demonstrated as follows [17]. Firstly a space $F$, namely

$$
\begin{gathered}
F=\left\{f(n)=f\left(n, t_{1}, t_{2}, \cdots, t_{j}, \cdots\right) ; n \in \mathbb{Z}, t_{i} \in \mathbb{R}\right\} \\
\text { Co-published by Atlantis Press and Taylor \& Francis } \\
\text { Copyright: the authors }
\end{gathered}
$$


is defined for the space of the dKP hierarchy. $\Lambda$ and $\Delta$ are denote for the shift operator and the difference operator, respectively. Their actions on function $f(n)$ are defined as

$$
\Lambda f(n)=f(n+1)
$$

and

$$
\Delta f(n)=f(n+1)-f(n)=(\Lambda-I) f(n)
$$

respectively, where $I$ is the identity operator.

For any $j \in \mathbb{Z}$, the Leibniz rule of $\Delta$ operation is,

$$
\Delta^{j} f=\sum_{i=0}^{\infty}\left(\begin{array}{l}
j \\
i
\end{array}\right)\left(\Delta^{i} f\right)(n+j-i) \Delta^{j-i}, \quad\left(\begin{array}{l}
j \\
i
\end{array}\right)=\frac{j(j-1) \cdots(j-i+1)}{i !}
$$

So an associative ring $F(\Delta)$ of formal pseudo difference operators (PDO) is obtained, namely $F(\Delta)=\left\{R=\sum_{j=-\infty}^{d} f_{j}(n) \Delta^{j}, f_{j}(n) \in R, n \in \mathbb{Z}\right\}$. The adjoint operator to the $\Delta$ operator is given by $\Delta^{*}$,

$$
\Delta^{*} f(n)=\left(\Lambda^{-1}-I\right) f(n)=f(n-1)-f(n)
$$

where $\Lambda^{-1} f(n)=f(n-1)$, and the corresponding $j$-times operation is

$$
\Delta^{* j} f=\sum_{i=0}^{\infty}\left(\begin{array}{l}
j \\
i
\end{array}\right)\left(\Delta^{* i} f\right)(n+i-j) \Delta^{* j-i}
$$

Then the adjoint ring $F\left(\Delta^{*}\right)$ to the $F(\Delta)$ is obtained, and the formal adjoint to $R \in F(\Delta)$ is defined by $R^{*} \in F\left(\Delta^{*}\right)$ as $R^{*}=\sum_{j=-\infty}^{d} \Delta^{* j} f_{j}(n)$. The " *" stands for the conjugate operation which satisfies the rules as $(F G)^{*}=G^{*} F^{*}, \Delta^{*}=-\Delta, f^{*}=f$ for two operators $F$ and $G$ and $f(n)^{*}=f(n)$ for a function $f(n)$. Here for any (pseudo-) difference operator $A$ and a function $f$, the symbol $A(f)$ will indicate the action of $A$ on $f$, whereas the symbol $A f$ (or $A \cdot f$ ) will denote just operator product of $A$ and $f$.

The dKP hierarchy $[17,23]$ is a family of evolution equations depending on infinitely many variables $t=\left(t_{1}, t_{2}, \cdots\right)$

$$
\frac{\partial L}{\partial t_{k}}=\left[B_{k}, L\right], \quad B_{k}:=\left(L^{k}\right)_{+}
$$

where $L$ is a general first-order PDO

$$
L(n)=\Delta+\sum_{j=1}^{\infty} f_{j}(n) \Delta^{-j}
$$

$B_{m}=\left(L^{m}\right)_{+}=\sum_{j=0}^{m} a_{j}(n) \Delta^{j}$, i. e. $\left(L^{m}\right)_{+}$is the non-negative projection of $L^{m}$, and $\left(L^{m}\right)_{-}=L^{m}-$ $\left(L^{m}\right)_{+}$is the negative projection of $L^{m}$. The Lax operator in eq.(2.8) can be generated by a dressing 
operator

$$
W(n ; t)=1+\sum_{j=1}^{\infty} w_{j}(n ; t) \Delta^{-j}
$$

through

$$
L=W \Delta W^{-1} .
$$

Further the flow equation (2.7) is equivalent to the so-called Sato equation,

$$
\frac{\partial W}{\partial t_{k}}=-\left(L^{k}\right)_{-} W
$$

If the functions $q(t)$ and $r(t)$ satisfy

$$
\frac{\partial q}{\partial t_{k}}=B_{k}(q), \quad \frac{\partial r}{\partial t_{k}}=-B_{k}^{*}(r),
$$

then we call them the eigenfunction and the adjoint eigenfunction respectively.

The cdKP hierarchy [28] is defined by restricting the Lax operator of the dKP hierarchy

$$
\frac{\partial L}{\partial t_{k}}=\left[B_{k}, L\right], \quad B_{k}:=\left(L^{k}\right)_{+},
$$

with the following $l$-constrained form:

$$
L^{l}=L_{+}^{l}+\sum_{i=1}^{m} q_{i} \Delta^{-1} r_{i}=\Delta^{l}+\sum_{j=0}^{k-2} v_{j} \Delta^{j}+\sum_{i=1}^{m} q_{i} \Delta^{-1} r_{i}
$$

where $q_{i}$ and $r_{i}$ are the eigenfunction and adjoint eigenfunction respectively.

\section{The Two Types Gauge Transformations Of The cdKP Hierarchy}

Let $L$ be the original Lax operator of the cdKP hierarchy (2.14), and $T$ be a pseduo-difference operator. If the transformation

$$
\widetilde{L}=T L T^{-1}
$$

such that

$$
\frac{\partial \widetilde{L}}{\partial t_{k}}=\left[\widetilde{B}_{k}, \widetilde{L}\right], \quad \widetilde{B}_{k}=\left(\widetilde{L}^{k}\right)_{+}, k=1,2,3, \cdots
$$

still holds for transformed Lax operator $\widetilde{L}$, then $T$ is called the gauge transformation operator of the cdKP hierarchy.

Similar to the KP hierarchy [3], there are two types of gauge transformation operators of the dKP hierarchy as $[30,35]$

$$
\begin{aligned}
\text { Type I }: & T_{D}(q)=\Lambda(q) \Delta q^{-1}, \\
\text { Type II }: & T_{I}(r)=\Lambda^{-1}\left(r^{-1}\right) \Delta^{-1} r,
\end{aligned}
$$

where $q$ and $r$ are the eigenfunction and adjoint eigenfunction respectively. The type I transformation is called the difference type, while the type II is called the integral type. 
Here we review some results about the integral type and the difference type gauge transformations of the cdKP hierarchy [27]. Under the integral type gauge transformation $T_{I}(r)$, the transformed Lax operator will be:

$$
\begin{aligned}
\widetilde{L} & =T_{I}(r) L T_{I}(r)^{-1}=\widetilde{L}_{+}+\widetilde{L}_{-}, \\
\widetilde{L}_{+} & =\Lambda^{-1}\left(L_{+}\right)-\Lambda^{-1}\left(r^{-1}\right) \Delta^{-1}\left(\Delta^{*}\left(r^{-1} L_{+}^{*} r\right)_{\geq 1}\right)^{*}(r), \\
\widetilde{L}_{-} & =\widetilde{q}_{0} \Delta^{-1} \widetilde{r}_{0}+\sum_{i=1}^{m} \widetilde{q}_{i} \Delta^{-1} \widetilde{r}_{i}, \\
\widetilde{q}_{0} & =\Lambda^{-1}\left(r^{-1}\right), \quad \widetilde{r}_{0}=T_{I}(r)^{*-1} L^{(0) *}(r), \\
\widetilde{q}_{i} & =T_{I}(r)\left(q_{i}\right), \quad \widetilde{r}_{i}=T_{I}(r)^{*-1}\left(r_{i}\right) .
\end{aligned}
$$

In order to preserve the form (2.14) of the Lax operator $L, r$ is required to coincide with one of the original adjoint eigenfunctions of $L$, e.g. $r=r_{1}$, since $\widetilde{r}_{1}=0$ in this case.

Under the gauge transformation of $T_{D}(q)$, the transformed Lax operator reads as

$$
\begin{aligned}
\widetilde{L} & =\widetilde{L}_{+}+\widetilde{L}_{-}, \\
\widetilde{L}_{+} & =\Lambda\left(L_{+}\right)+\Lambda(q) \Delta\left(q^{-1} L_{+} q\right)_{\geq 1} \Delta^{-1} \Lambda\left(q^{-1}\right), \\
\widetilde{L}_{-} & =\widetilde{q}_{0} \Delta^{-1} \widetilde{r}_{0}+\sum_{i=1}^{m} \widetilde{q}_{i} \Delta^{-1} \widetilde{r}_{i}, \\
\widetilde{q}_{0} & =T_{D}(q)(L)(q), \widetilde{r}_{0}=\Lambda\left(q^{-1}\right), \\
\widetilde{q}_{i} & =T_{D}(q) q_{i}, \widetilde{r}_{i}=\left(T_{D}^{-1}\right)^{*}(q)\left(r_{i}\right) .
\end{aligned}
$$

For the difference type gauge transformation $T_{D}(q)$, in order to preserve the form (2.14) of the Lax operator $L, q$ is required to coincide with one of the original adjoint eigenfunctions of $L$, e.g. $q=q_{1}$, since $\widetilde{q}_{1}=0$ in this case.

In order to calculate the transformed formula of the part as $f \Delta^{-1} g$ in the Lax operator under the integral type gauge transformation, the following lemma is necessary.

\section{Lemma 3.1.}

$$
\begin{aligned}
T_{I}\left(r_{a}\right) \cdot M \Delta^{-1} r_{a} \cdot T_{I}^{-1}\left(r_{a}\right)= & \Lambda^{-1}\left(r_{a}^{-1}\right) \Delta^{-1}\left\{T_{I}^{*-1}\left(r_{a}\right)\left(M \Delta^{-1} r_{a}\right)^{*}\left(r_{a}\right)\right\} \\
T_{I}\left(r_{a}\right) \cdot q_{a} \Delta^{-1} N \cdot T_{I}\left(r_{a}\right)^{-1}= & \Lambda^{-1}\left(r_{a}^{-1}\right) r_{a}^{-1} \Delta^{-1}\left\{T_{I}^{*-1}\left(r_{a}\right)\left(q_{a} \Delta^{-1} N\right)^{*}\left(r_{a}\right)\right\} \\
& +\widetilde{L}\left(\widetilde{q}_{a}\right) \Delta^{-1} \widetilde{N} \\
T_{I}\left(r_{a}\right) \cdot M \Delta^{-1} N \cdot T_{I}\left(r_{a}\right)^{-1}= & \Lambda^{-1}\left(r_{a}^{-1}\right) \Delta^{-1}\left\{T_{I}^{*-1}\left(r_{a}\right)\left(M \Delta^{-1} N\right)^{*}\left(r_{a}\right)\right\} \\
& +\widetilde{M} \Delta^{-1} \widetilde{N}, \\
\widetilde{L}^{k+1}\left(\widetilde{q}_{a}\right)= & T_{I}\left(r_{a}\right) L^{k}\left(q_{a}\right), \quad k=0,1,2, \ldots, \\
\left(\widetilde{L}^{*}\right)^{k-1}\left(\widetilde{r}_{a}\right)= & T_{I}\left(r_{a}\right)^{*-1}\left(L^{*}\right)^{k}\left(r_{a}\right), \quad k=1,2,3 \ldots
\end{aligned}
$$

where $r_{a}$ is one of the adjoint eigenfunctions of the cdKP hierarchy (2.14), $M$ and $N$ are two functions of $t$, and

$$
\begin{aligned}
& \widetilde{L}=T_{I}\left(r_{a}\right) L T_{I}\left(r_{a}\right)^{-1}, \widetilde{q}_{a}=\Lambda^{-1}\left(1 / r_{a}\right), \widetilde{r}_{a}=T_{I}\left(r_{a}\right)^{*-1} L^{*}\left(r_{a}\right), \\
& \widetilde{M}=T_{I}\left(r_{a}\right)(M), \widetilde{N}=T_{I}\left(r_{a}\right)^{*-1}(N) .
\end{aligned}
$$


Proof. Firstly, according to $\Delta^{-1} f \Delta^{-1}=\left(\Delta^{-1} f\right) \Delta^{-1}-\Delta^{-1} \Lambda\left(\Delta^{-1} f\right)$ and $\Delta f-\Lambda(f) \Delta=\Delta(f)$,

$$
\begin{aligned}
& T_{I}\left(r_{a}\right) \cdot M \Delta^{-1} N \cdot T_{I}\left(r_{a}\right)^{-1}=\Lambda^{-1}\left(r_{a}^{-1}\right) \Delta^{-1} r_{a} \cdot M \Delta^{-1} N \cdot r_{a}^{-1} \Delta \Lambda^{-1}\left(r_{a}\right) \\
= & \Lambda^{-1}\left(r_{a}^{-1}\right)\left(\Delta^{-1}\left(r_{a} M\right) \Delta^{-1}-\Delta^{-1} \Lambda\left(\Delta^{-1}\left(r_{a} M\right)\right)\right) N r_{a}^{-1} \Delta \Lambda^{-1}\left(r_{a}\right) \\
= & \Lambda^{-1}\left(r_{a}^{-1}\right) \Delta^{-1}\left(r_{a} M\right) \Delta^{-1}\left(\Delta \Lambda^{-1}\left(N r_{a}^{-1}\right)-\Delta \Lambda^{-1}\left(N r_{a}^{-1}\right)\right) \Lambda^{-1}\left(r_{a}\right) \\
& -\Lambda^{-1}\left(r_{a}^{-1}\right) \Delta^{-1}\left(\Delta \Lambda^{-1}\left(\Lambda\left(\Delta^{-1}\left(r_{a} M\right)\right) N r_{a}^{-1}\right)-\Delta\left(\Delta^{-1}\left(r_{a} M\right) \Lambda^{-1}\left(N r_{a}^{-1}\right)\right)\right) \Lambda^{-1}\left(r_{a}\right) \\
= & \Lambda^{-1}\left(r_{a}^{-1}\right) \Delta^{-1}\left(r_{a} M\right) \Lambda^{-1}(N)-\Lambda^{-1}\left(r_{a}^{-1}\right) \Delta^{-1}\left(r_{a} M\right) \Delta^{-1}\left(\Delta \Lambda^{-1}\left(N r_{a}^{-1}\right)\right) \Lambda^{-1}\left(r_{a}\right) \\
& -\Lambda^{-1}\left(r_{a}^{-1}\right) \Delta^{-1}\left(r_{a} M\right) \Lambda^{-1}(N)+\Lambda^{-1}\left(r_{a}^{-1}\right) \Delta^{-1} \Delta\left(\Delta^{-1}\left(r_{a} M\right) \Lambda^{-1}\left(N r_{a}^{-1}\right)\right) \Lambda^{-1}\left(r_{a}\right) \\
= & T_{I}\left(r_{a}\right)(M) \Delta^{-1} T_{I}\left(r_{a}\right)^{*-1}(N)+\Lambda^{-1}\left(r_{a}^{-1}\right) \Delta^{-1}\left\{T_{I}\left(r_{a}\right)^{*-1}\left(M \Delta^{-1} N\right)^{*}\left(r_{a}\right)\right\} \\
= & \widetilde{M} \Delta^{-1} \widetilde{N}+\Lambda^{-1}\left(r_{a}^{-1}\right) \Delta^{-1}\left\{T_{I}\left(r_{a}\right)^{*-1}\left(M \Delta^{-1} N\right)^{*}\left(r_{a}\right)\right\} .
\end{aligned}
$$

So (3.17) is be proved. (3.15) can be derived from (3.17) for $N=r_{a}$, since $T_{I}\left(r_{a}\right)^{*-1}\left(r_{a}\right)=0$.

Then for (3.18),

$$
\begin{aligned}
\widetilde{L}^{k+1}\left(\widetilde{q}_{a}\right) & =T_{I}\left(r_{a}\right) L^{k+1} T_{I}\left(r_{a}\right)^{-1}\left(\Lambda^{-1}\left(r_{a}^{-1}\right)\right) \\
& =T_{I}\left(r_{a}\right) L^{k}\left(L_{+}+\sum_{i=0}^{k} q_{i} \Delta^{-1} r_{i}\right) r_{a}^{-1} \Delta \Lambda^{-1}\left(r_{a}\right) \Lambda^{-1}\left(r_{a}^{-1}\right)=T_{I}\left(r_{a}\right) L^{k}\left(q_{a}\right) .
\end{aligned}
$$

Here we let $q_{i} \Delta^{-1}(0)=0$ for $i \neq a$, and $q_{a} \Delta^{-1}(0)=q_{a}$. And (3.16) can be derived from (3.17) and (3.18). At last,

$$
T_{I}\left(r_{a}\right)^{*-1}\left(L^{*}\right)^{k}\left(r_{a}\right)=T_{I}\left(r_{a}\right)^{*-1}\left(L^{*}\right)^{k-1} T_{I}\left(r_{a}\right)^{*} T_{I}\left(r_{a}\right)^{*-1} L^{*}\left(r_{a}\right)=\left(\widetilde{L}^{*}\right)^{k-1}\left(\widetilde{r}_{a}\right) .
$$

In order to calculate the transformed formula of the part as $f \Delta^{-1} g$ in the Lax operator under the difference type gauge transformation, the following lemma is necessary.

\section{Lemma 3.2.}

$$
\begin{aligned}
T_{D}\left(q_{a}\right) \cdot M \Delta^{-1} r_{a} \cdot T_{D}\left(q_{a}\right)^{-1} & =T_{D}\left(q_{a}\right)\left(M \Delta^{-1} r_{a}\right)\left(q_{a}\right) \Delta^{-1} \Lambda\left(q_{a}^{-1}\right)+\widetilde{M} \Delta^{-1} \widetilde{L}^{*}\left(\widetilde{r}_{a}\right), \\
T_{D}\left(q_{a}\right) \cdot q_{a} \Delta^{-1} N \cdot T_{D}\left(q_{a}\right)^{-1} & =T_{D}\left(q_{a}\right)\left(q_{a} \Delta^{-1} N\right)\left(q_{a}\right) \Delta^{-1} \Lambda\left(q_{a}^{-1}\right), \\
T_{D}\left(q_{a}\right) \cdot M \Delta^{-1} N \cdot T_{D}\left(q_{a}\right)^{-1} & =T_{D}\left(q_{a}\right)\left(M \Delta^{-1} N\right)\left(q_{a}\right) \Delta^{-1} \Lambda\left(q_{a}^{-1}\right)+\widetilde{M} \Delta^{-1} \widetilde{N} \\
\widetilde{L}^{k-1}\left(\widetilde{q}_{a}\right) & =T_{D}\left(q_{a}\right) L^{k}\left(q_{a}\right), \quad k=0,1,2, \ldots, \\
\left(\widetilde{L}^{*}\right)^{k}\left(\widetilde{r}_{a}\right) & =T_{D}^{*}\left(q_{a}\right)^{-1}\left(L^{*}\right)^{k-1}\left(r_{a}\right), \quad k=1,2,3, \ldots,
\end{aligned}
$$

where $r_{a}$ is one of the adjoint eigenfunctions of the cdKP hierarchy (2.14), $M$ and $N$ are two functions of $t$, and

$$
\begin{array}{r}
\widetilde{L}=T_{D}\left(q_{a}\right) L T_{D}\left(q_{a}\right)^{-1}, \widetilde{q}_{a}=T_{D}\left(q_{a}\right) L\left(q_{a}\right), \widetilde{r}_{a}=\Lambda\left(r_{a}^{-1}\right), \\
\widetilde{M}=T_{D}\left(q_{a}\right)(M), \widetilde{N}=T_{D}\left(q_{a}\right)^{*-1}(N) .
\end{array}
$$


Proof. Firstly, according to $\Delta^{-1} f \Delta^{-1}=\left(\Delta^{-1} f\right) \Delta^{-1}-\Delta^{-1} \Lambda\left(\Delta^{-1} f\right)$ and $\Delta f-\Lambda(f) \Delta=\Delta(f)$,

$$
\begin{aligned}
& T_{D}\left(q_{a}\right) \cdot M \Delta^{-1} N \cdot T_{D}\left(q_{a}\right)^{-1}=\Lambda\left(q_{a}\right) \Delta q_{a}^{-1} \cdot M \Delta^{-1} N \cdot q_{a} \Delta^{-1} \Lambda\left(q_{a}^{-1}\right) \\
= & \Lambda\left(q_{a}\right) \Delta\left(q_{a}^{-1} M\right)\left(\Delta^{-1}\left(N q_{a}\right) \Delta^{-1}-\Delta^{-1} \Lambda\left(\Delta^{-1}\left(q_{a} N\right)\right)\right) \Lambda\left(q_{a}^{-1}\right) \\
= & \Lambda\left(q_{a}\right)\left(\Delta\left(q_{a}^{-1} M \Delta^{-1}\left(N q_{a}\right)\right)+\Lambda\left(q_{a}^{-1} M \Delta^{-1}\left(N q_{a}^{-1}\right)\right) \Delta\right) \Delta^{-1} \Lambda\left(q_{a}^{-1}\right) \\
& -\Lambda\left(q_{a}\right)\left(\Delta\left(q_{a}^{-1} M\right)+\Lambda\left(q_{a}^{-1} M\right) \Delta\right) \Delta^{-1} \Lambda\left(\Delta^{-1}\left(N q_{a}\right)\right) \Lambda^{-1}\left(q_{a}^{-1}\right) \\
= & \Lambda\left(q_{a}\right) \Delta\left(q_{a}^{-1} M \Delta^{-1}\left(N q_{a}\right)\right) \Delta^{-1} \Lambda\left(q_{a}^{-1}\right)+\Lambda\left(q_{a}\right) \Lambda\left(q_{a}^{-1} M \Delta^{-1}\left(N q_{a}\right)\right) \Lambda\left(q_{a}^{-1}\right) \\
& -\Lambda\left(q_{a}\right) \Delta\left(q_{a}^{-1} M\right) \Delta^{-1} \Lambda\left(\Delta^{-1}\left(N q_{a}\right)\right) \Lambda\left(q_{a}^{-1}\right)-\Lambda\left(q_{a}\right) \Lambda\left(q_{a}^{-1} M \Delta^{-1}\left(N q_{a}\right)\right) \Lambda\left(q_{a}^{-1}\right) \\
= & \Lambda\left(q_{a}\right) \Delta\left(q_{a}^{-1} M \Delta^{-1}\left(N q_{a}\right)\right) \Delta^{-1} \Lambda\left(q_{a}^{-1}\right)-\Lambda\left(q_{a}\right) \Delta\left(q_{a}^{-1} M\right) \Delta^{-1} \cdot \Lambda \Delta^{-1}\left(N q_{a}\right) \Lambda\left(q_{a}^{-1}\right) \\
= & T_{D}\left(q_{a}\right)(M) \Delta^{-1} T_{D}\left(q_{a}\right)^{*-1}(N)+T_{D}\left(q_{a}\right)\left(M \Delta^{-1} N\right)\left(q_{a}\right) \Delta^{-1} \Lambda\left(q_{a}^{-1}\right) \\
= & \widetilde{M} \Delta^{-1} \widetilde{N}+T_{D}\left(q_{a}\right)\left(M \Delta^{-1} N\right)\left(q_{a}\right) \Delta^{-1} \Lambda\left(q_{a}^{-1}\right) .
\end{aligned}
$$

So (3.23) is be proved. (3.22) can be derived from (3.23) for $M=q_{a}$, since $T_{D}\left(q_{a}\right)\left(q_{a}\right)=0$.

For (3.24),

$$
\widetilde{L}^{k-1}\left(\widetilde{q}_{a}\right)=T_{D}\left(q_{a}\right) L^{k-1} T_{D}\left(q_{a}\right)^{-1} T_{D}\left(q_{a}\right) L\left(q_{a}^{-1}\right)=T_{D}\left(q_{a}\right) L^{k}\left(q_{a}\right) .
$$

Then (3.21) can be derived from (3.23) and (3.25).

At last, for (3.25),

$$
T_{D}^{*}\left(q_{a}\right)^{-1}\left(L^{*}\right)^{k+1}\left(r_{a}\right)=T_{D}^{*}\left(q_{a}\right)^{-1}\left(L^{*}\right)^{k} T_{D}^{*}\left(q_{a}\right) T_{D}^{*}\left(q_{a}\right)^{-1} L^{*}\left(r_{a}\right)=\left(\widetilde{L}^{*}\right)^{k}\left(\widetilde{r}_{a}\right) .
$$

In order to prove the compatibility between the two types of the gauge transformation and the additional symmetry, the following operator identities are necessary.

Lemma 3.3. Let $q, r$ be suitable function and $A$ be a PDO, then

$$
\begin{aligned}
\left(\Lambda^{-1}\left(r^{-1}\right) \Delta^{-1} r A r^{-1} \Delta \Lambda^{-1}(r)\right)_{-}= & \Lambda^{-1}\left(r^{-1}\right) \Delta^{-1} r A_{-} r^{-1} \Delta \Lambda^{-1}(r) \\
& -\Lambda^{-1}\left(r^{-1}\right) \Delta^{-1} \Lambda^{-1}(r) \Delta\left(\Lambda^{-1}\left(r^{-1} A_{+}^{*} r\right)\right), \\
\left(\Lambda(q) \Delta q^{-1} A q \Delta^{-1} \Lambda\left(q^{-1}\right)\right)_{+}= & \Lambda(q) \Delta q^{-1} A_{+} q \Delta^{-1} \Lambda\left(q^{-1}\right) \\
& -\Lambda(q) \Delta\left(q^{-1} A_{+}(q)\right) \Delta^{-1} \Lambda\left(q^{-1}\right) .
\end{aligned}
$$

Proof. With $\left(K q \Delta^{-1} r\right)_{-}=K(q) \Delta^{-1} r,\left(q \Delta^{-1} r K\right)_{-}=q \Delta^{-1} K^{*}(r)$ for pure-difference operator $K$ [28],

$$
\begin{aligned}
& \left(\Lambda^{-1}\left(r^{-1}\right) \Delta^{-1} r A r^{-1} \Delta \Lambda^{-1}(r)\right)_{-} \\
= & \left(\Lambda^{-1}\left(r^{-1}\right) \Delta^{-1} r A_{-} r^{-1} \Delta \Lambda^{-1}(r)\right)_{-}+\left(\Lambda^{-1}\left(r^{-1}\right) \Delta^{-1} r A_{+} r^{-1} \Delta \Lambda^{-1}(r)\right)_{-} \\
= & \Lambda^{-1}\left(r^{-1}\right) \Delta^{-1} r A_{-} r^{-1} \Delta \Lambda^{-1}(r)-\Lambda^{-1}\left(r^{-1}\right) \Delta^{-1} \Lambda^{-1}(r) \Delta\left(\Lambda^{-1}\left(r^{-1} A_{+}^{*} r\right)\right),
\end{aligned}
$$


so the (3.27) is proved. For (3.28), with $\left(K q \Delta^{-1} r\right)_{-}=K(q) \Delta^{-1} r$,

$$
\begin{aligned}
\left(\Lambda(q) \Delta q^{-1} A q \Delta^{-1} \Lambda\left(q^{-1}\right)\right)_{+} & =\left(\Lambda(q) \Delta q^{-1} A_{+} q \Delta^{-1} \Lambda\left(q^{-1}\right)\right)_{+} \\
& =\Lambda(q) \Delta q^{-1} A_{+} q \Delta^{-1} \Lambda\left(q^{-1}\right)-\Lambda(q) \Delta\left(q^{-1} A_{+}(q)\right) \Delta^{-1} \Lambda\left(q^{-1}\right) .
\end{aligned}
$$

Remark 1: This lemma is a difference-analogue of the corresponding identities of PDO given by $[2,37]$.

\section{Additional Symmetries Of The cdKP Hierarchy}

Define

$$
X_{k}^{(1)}=\sum_{i=1}^{m} \sum_{j=0}^{k-1}\left(j-\frac{1}{2}(k-1)\right) L^{k-1-j}\left(q_{i}\right) \Delta^{-1}\left(L^{*}\right)^{j}\left(r_{i}\right) ; k \geq 1
$$

which is the essential to ensure the compatibility of the additional Virasoro symmetry with the constraints (2.14) defining the cdKP hierarchy. The additional symmetry flows for the cdKP hierarchy, spanning the Virasoro algebra, are given by [28]:

$$
\partial_{k}^{*} L=\left[-\left(M_{\Delta} L^{k}\right)_{-}+X_{k-1}^{(1)}, L\right] .
$$

$M_{\Delta}$ is the Orlov-Schulman operator [38] defined in the dressing the "bare" $M^{(0)}$ operator:

$$
M^{(0)}=\sum_{l \geq 1} l t_{l} \Delta^{l-k}=X_{(k)}+\sum_{l \geq 1}(l+k) t_{l+k} \Delta^{l} ; X_{(k)}=\sum_{l=1}^{k} l t_{l} \Delta^{l-k}
$$

that is,

$$
\begin{aligned}
M_{\Delta} & =W M^{(0)} W^{-1}=W X_{(k)} W^{-1}+\sum_{l \geq 1}(l+k) t_{l+k} L^{l}=\sum_{l \geq 0}(l+k) t_{l+k} L_{+}^{l}+\left(M_{\Delta}\right)_{-}, \\
\left(M_{\Delta}\right)_{-} & =W X_{(k)} W^{-1}-k t_{k}-\sum_{l \geq 1}(l+k) t_{l+k} \frac{\partial W}{\partial t_{l}} W^{-1}
\end{aligned}
$$

with (2.11) used in (4.5).

Then accordingly, the actions of the additional symmetry flows on the dressing operators and BA functions are showed that:

$$
\partial_{k}^{*} W=\left(-\left(M_{\Delta} L^{k}\right)_{-}+X_{k-1}^{(1)}\right) W ; \partial_{k}^{*} \psi(t, \lambda)=\left(-\left(M_{\Delta} L^{k}\right)_{-}+X_{k-1}^{(1)}\right)(\psi(t, \lambda)) .
$$

The corresponding actions on the eigenfunctions $q_{i}$ and the adjoint eigenfunctions $r_{i}$ are derived by considering $\left(\partial_{k}^{*} L\right)_{-}$listed as follows [28]:

$$
\begin{aligned}
& \partial_{k}^{*} q_{i}=\left(M_{\Delta} L^{k}\right)_{+}\left(q_{i}\right)+\frac{k}{2} L^{k-1}\left(q_{i}\right)+X_{k-1}^{(1)}\left(q_{i}\right), \\
& \partial_{k}^{*} r_{i}=-\left(M_{\Delta} L^{k}\right)_{+}^{*}\left(r_{i}\right)+\frac{k}{2}\left(L^{*}\right)^{k-1}\left(r_{i}\right)-\left(X_{k-1}^{(1)}\right)^{*}\left(r_{i}\right) .
\end{aligned}
$$


M.H. Li, J.P. Cheng and J.S. He / Constrained discrete Kadomtsev-Petviashvili hierarchy

\section{Additional Symmetries Versus Two Types Gauge Transformations}

In this section, we will restrict to the cdKP hierarchy $((2.14)$ for $m=1, l=1)$. And thus its Lax operator is given by

$$
L=\Delta+q \Delta^{-1} r
$$

In order to investigate the changes of the additional symmetries under the integral type gauge transformation $T_{I}(r)$, some useful lemmas are needed.

\section{Lemma 5.1.}

$$
\begin{aligned}
T_{I}(r) X_{k-1}^{(1)} T_{I}(r)^{-1}= & \widetilde{X}_{k-1}^{(1)}+\sum_{j=0}^{k-2} \widetilde{L}^{k-j-2}(\widetilde{q}) \Delta^{-1} \widetilde{L}^{j}(\widetilde{r}) \\
& +\Lambda\left(r^{-1}\right) \Delta^{-1}\left\{\left(T_{I}^{*}(r)\right)^{-1}\left(X_{k-1}^{(1)}-\frac{k}{2} L^{k-1}\right)^{*}(r)\right\}
\end{aligned}
$$

Proof. According to Lemma 3.1 and (4.1), then

$$
\begin{aligned}
& T_{I}(r) X_{k-1}^{(1)} T_{I}(r)^{-1} \\
= & -\Lambda^{-1}\left(r^{-1}\right) \Delta^{-1} T_{D}(r)\left(X_{k-1}^{(1)}\right)^{*}(r)+\sum_{j=1}^{k-2}\left(j-\frac{1}{2}(k-2)\right) \widetilde{L}^{k-j-1}(\widetilde{q}) \Delta^{-1}\left(\widetilde{L}^{*}\right)^{j-1}(\widetilde{r}) \\
= & -\Lambda^{-1}\left(r^{-1}\right) \Delta^{-1} T_{D}(r)\left(X_{k-1}^{(1)}\right)^{*}(r)+\sum_{j=0}^{k-2}\left(j-\frac{1}{2}(k-2)\right) \widetilde{L}^{k-j-2}(\widetilde{q}) \Delta^{-1}\left(\widetilde{L}^{*}\right)^{j}(\widetilde{r}) \\
& +\sum_{j=0}^{k-2} \widetilde{L}^{k-j-2}(\widetilde{q}) \Delta^{-1}\left(\widetilde{L}^{*}\right)^{j}(\widetilde{r})-\left(1+k-2-\frac{1}{2}(k-2)\right) \widetilde{q} \Delta^{-1}\left(\widetilde{L}^{*}\right)^{k-2}(\widetilde{r}) \\
= & -\Lambda^{-1}\left(r^{-1}\right) \Delta^{-1} T_{D}(r)\left(X_{k-1}^{(1)}\right)^{*}(r)+\widetilde{X}_{k-1}^{(1)}+\sum_{j=0}^{k-2} \widetilde{L}^{k-j-2}(\widetilde{q}) \Delta^{-1}\left(\widetilde{L}^{*}\right)^{j}(\widetilde{r}) \\
& -\frac{k}{2} r^{-1} \Delta^{-1} T_{I}(r)^{*-1}\left(L^{*}\right)^{k-1}(r) \\
= & \widetilde{X}_{k-1}^{(1)}+\sum_{j=0}^{k-2} \widetilde{L}^{k-j-2}(\widetilde{q}) \Delta^{-1} \widetilde{L}^{j}(\widetilde{r})+r^{-1} \Delta^{-1}\left\{T_{I}(r)^{*-1}\left(X_{k-1}^{(1)}-\frac{k}{2} L^{k-1}\right)^{*}(r)\right\} .
\end{aligned}
$$

\section{Lemma 5.2.}

$$
\partial_{k}^{*} T_{I}(r) \cdot T_{I}(r)^{-1}=\Lambda^{-1}\left(r^{-1}\right) \Delta^{-1}\left\{T_{I}(r)^{*-1}\left(-\left(M_{\Delta} L^{k}\right)_{+}^{*}+\frac{k}{2}\left(L^{*}\right)^{k-1}-\left(X_{k-1}^{(1)}\right)^{*}\right)(r)\right\} .
$$


Proof. By (4.8),

$$
\begin{aligned}
\partial_{k}^{*} T_{I}(r) \cdot T_{I}(r)^{-1} & =-T_{I}(r) \partial_{k}^{*}\left(T_{I}(r)^{-1}\right) \\
& =\Lambda^{-1}\left(r^{-1}\right) \Delta^{-1} r^{-1} \partial_{k}^{*}(r) \Delta \Lambda^{-1}(r)-\Lambda^{-1}\left(r^{-1} \partial_{k}^{*}(r)\right) \\
& =\Lambda^{-1}\left(r^{-1}\right) \Delta^{-1}\left(\Delta \Lambda^{-1}\left(\partial_{k}^{*}(r) r^{-1}\right)-\Delta \Lambda^{-1}\left(\partial_{k}^{*}(r) r^{-1}\right)\right) \Lambda^{-1}(r)-\Lambda^{-1}\left(r^{-1} \partial_{k}^{*}(r)\right) \\
& =-\Lambda^{-1}\left(r^{-1}\right) \Delta^{-1}\left(\Delta \Lambda^{-1}\left(\partial_{k}^{*}(r) r^{-1}\right)\right) \Lambda^{-1}(r) \\
& =\Lambda^{-1}\left(r^{-1}\right) \Delta^{-1} T_{I}(r)^{*-1} \Lambda^{-1}\left(\partial_{k}^{*} r\right) \\
& =\Lambda^{-1}\left(r^{-1}\right) \Delta^{-1}\left\{T_{I}(r)^{*-1}\left(-\left(M_{\Delta} L^{k}\right)_{+}^{*}+\frac{k}{2}\left(L^{*}\right)^{k-1}-\left(X_{k-1}^{(1)}\right)^{*}\right)(r)\right\} .
\end{aligned}
$$

Theorem 5.1. The additional symmetry flows (4.2) for the cdKP hierarchy $((2.14)$ for $m=1, l=1)$ commute with the integral type transformations $T_{I}(r)$ preserving the form of $c d K P$ hierarchy, up to shifting of (4.2) by ordinary time flows, that is,

$$
\partial_{k}^{*} \widetilde{L}=\left[-\left(\widetilde{M}_{\Delta} \widetilde{L}^{k}\right)_{-}+\widetilde{X}_{k-1}^{(1)}, \widetilde{L}\right]-\frac{\partial \widetilde{L}}{\partial t_{k-1}} .
$$

Proof. Firstly, by (4.2),

$$
\begin{aligned}
\partial_{k}^{*} \widetilde{L} & =\partial_{k}^{*} T_{I}(r) \cdot L T_{I}(r)^{-1}+T_{I}(r) \partial_{k}^{*} L \cdot T_{I}(r)^{-1}-T_{I}(r) L T_{I}(r)^{-1} \cdot \partial_{k}^{*} T_{I}(r) \cdot T_{I}(r)^{-1} \\
& =\left[T_{I}(r)\left(-\left(M_{\Delta} L^{k}\right)_{-}+X_{k-1}^{(1)}\right) T_{I}(r)^{-1}+\partial_{k}^{*} T_{I}(r) \cdot T_{I}(r)^{-1}, \widetilde{L}\right]
\end{aligned}
$$

Then with the help of (5.2), (5.3), and the following useful formula in Lemma.3.3, we have

$$
\begin{aligned}
& T_{I}(r)\left(-\left(M_{\Delta} L^{k}\right)_{-}+X_{k-1}^{(1)}\right) T_{I}(r)^{-1}+\partial_{k}^{*} T_{I}(r) \cdot T_{I}(r)^{-1} \\
= & \left.T_{I}(r)\left(-M_{\Delta} L^{k}\right)_{-}\right) T_{I}^{-1}(r)-\Lambda^{-1}\left(r^{-1}\right) \Delta^{-1}\left\{T_{I}^{*}(r)^{-1}\left(\left(M_{\Delta} L^{k}\right)_{+}^{*}-\frac{k}{2}\left(L^{*}\right)^{k-1}+\left(X_{k-1}^{(1)}\right)^{*}\right)(r)\right\} \\
& +\widetilde{X}_{k-1}^{(1)}+\sum_{j=0}^{k-2} \widetilde{L}^{k-j-2}(\widetilde{q}) \Delta^{-1} \widetilde{L}^{j}(\widetilde{r})+\Lambda^{-1}\left(r^{-1}\right) \Delta^{-1}\left\{T_{I}(r)^{*-1}\left(\left(X_{k-1}^{(1)}\right)^{*}-\frac{k}{2}\left(L^{*}\right)^{k-1}\right)(r)\right\} \\
= & -\left(\widetilde{M}_{\Delta} \widetilde{L}^{k}\right)_{-}+\widetilde{X}_{k-1}^{(1)}+\left(\widetilde{L}^{k-1}\right)_{-},
\end{aligned}
$$

where the following relation [28] is used,

$$
\left(\widetilde{L}^{k-1}\right)_{-}=\sum_{j=0}^{k-2} \widetilde{L}^{k-j-2}(\widetilde{q}) \Delta^{-1} \widetilde{L}^{j}(\widetilde{r}) .
$$

In the above process,

$$
\begin{aligned}
& -T_{I}(r)\left(M_{\Delta} L^{k}\right)_{-} T_{I}^{-1}(r)-\Lambda^{-1}\left(r^{-1}\right) \Delta^{-1} T_{I}^{*}(r)^{-1}\left(\left(M_{\Delta} L^{k}\right)_{+}^{*}(r)\right) \\
& \left.=-T_{I}(r)\left(M_{\Delta} L^{k}\right)_{-} T_{I}^{-1}(r)+\Lambda^{-1}\left(r^{-1}\right) \Delta^{-1}\left(r\left(M_{\Delta} L^{k}\right)_{+} T_{I}(r)^{-1}\right)^{*}\right) \\
& =-\left(\widetilde{M}_{\Delta} \widetilde{L}^{k}\right)_{-},
\end{aligned}
$$

which can be got by means of the identity (3.27) of Lemma.3.3.

At last, the substituting (5.6) into (5.5) gives rise to (5.4). 
For the difference type gauge transformation $T_{D}(q)$, there are some lemma as following.

\section{Lemma 5.3.}

$$
T_{D}(q) X_{k-1}^{(1)} T_{D}^{-1}(q)=\widetilde{X}_{k-1}^{(1)}-\left(\widetilde{L}^{k-1}\right)_{-}+\left(T_{D}(q)\left(X_{k-1}^{(1)}+\frac{k}{2} L^{k-1}\left(q_{i}\right)\right)\right) q \Delta^{-1} \Lambda\left(q^{-1}\right) .
$$

Proof. According to Lemma 3.2 and (4.1), then

$$
\begin{aligned}
& T_{D}(q) X_{k-1}^{(1)} T_{D}(q)^{-1} \\
= & \Lambda(q) \Delta q^{-1} \sum_{j=0}^{k-2}\left(j-\frac{k-2}{2}\right) L^{k-j-2}\left(q_{i}\right) \Delta^{-1}\left(L^{*}\right)^{j}\left(r_{i}\right) q \Delta^{-1} \Lambda\left(q^{-1}\right) \\
\stackrel{(3.23)}{=} & \sum_{j=0}^{k-2}\left(j-\frac{k-2}{2}\right) T_{D}(q)\left(L^{k-2}\left(q_{i}\right) \Delta^{-1} r_{i}\right)(q) \Delta^{-1} \Lambda\left(q^{-1}\right)+\widetilde{L}^{k-j-3}\left(\widetilde{q}_{i}\right) \Delta^{-1}\left(\widetilde{L}^{*}\right)^{j+1}\left(\widetilde{r}_{i}\right) \\
= & \sum_{j=0}^{k-2}\left(j-\frac{k-2}{2}\right) T_{D}(q)\left(L^{k-2}\left(q_{i}\right) \Delta^{-1} r_{i}\right)(q) \Delta^{-1} \Lambda\left(q^{-1}\right)+\sum_{j=1}^{k-1}\left(j-\frac{k}{2}\right) \widetilde{L}^{k-j-2}\left(\widetilde{q}_{i}\right) \Delta^{-1}\left(\widetilde{L}^{*}\right)^{j}\left(\widetilde{r}_{i}\right) \\
= & T_{D}(q)\left(X_{k-1}^{(1)}\right)(q) \Delta^{-1} \Lambda\left(q^{-1}\right)+\sum_{j=1}^{k-1}\left(j-\frac{k-2}{2}\right) \widetilde{L}^{k-j-2}\left(\widetilde{q}_{i}\right) \Delta^{-1}\left(\widetilde{L}^{*}\right)^{j}\left(\widetilde{r}_{i}\right) \\
& -\sum_{j=1}^{k-1} \widetilde{L}^{k-j-2}\left(\widetilde{q}_{i}\right) \Delta^{-1}\left(\widetilde{L}^{*}\right)^{j}\left(\widetilde{r}_{i}\right) \\
= & \sum_{j=0}^{k-2}\left(j-\frac{k-2}{2}\right) \widetilde{L}^{k-j-2}\left(\widetilde{q}_{i}\right) \Delta^{-1}\left(\widetilde{L}^{*}\right)^{j}\left(\widetilde{r}_{i}\right)-\sum_{j=0}^{k-2} \widetilde{L}^{k-j-2}\left(\widetilde{q}_{i}\right) \Delta^{-1}\left(\widetilde{L}^{*}\right)^{j}\left(\widetilde{r}_{i}\right) \\
& +T_{D}(q)\left(X_{k-1}^{(1)}\right)(q) \Delta^{-1} \Lambda\left(q^{-1}\right)+\frac{k}{2} T_{D}(q)\left(L^{k-2}\left(q_{i}\right) \Delta^{-1}\left(\widetilde{L}^{*}\right)^{0} r_{i}\right)(q) \Delta^{-1} \Lambda\left(q^{-1}\right) \\
= & \widetilde{X}_{k-1}^{(1)}-\left(\widetilde{L}^{k-1}\right)-+\left(T_{D}(q)\left(X_{k-1}^{(1)}+\frac{k}{2} L^{k-1}\left(q_{i}\right)\right)\right)(q) \Delta^{-1} \Lambda\left(q^{-1}\right) .
\end{aligned}
$$

Here we use the relation of $T_{D}\left(q_{a}\right)\left(q_{a}\right)=0$.

\section{Lemma 5.4.}

$$
\partial_{k}^{*} T_{D}(q) \cdot T_{D}^{-1}(q)=-\Lambda(q) \Delta\left(q^{-1}\left(\left(M_{\Delta} L^{k}\right)_{+}+\frac{k}{2} L^{k-1}+X_{k-1}^{(1)}\right)\right)(q) \Delta^{-1} \Lambda\left(q^{-1}\right) .
$$

Proof. By (4.7),

$$
\begin{aligned}
\partial_{k}^{*} T_{D}(q) \cdot T_{D}(q)^{-1} & =-T_{D}(q) \partial_{k}^{*}\left(T_{D}(q)^{-1}\right) \\
& =-\Lambda(q) \Delta q^{-1} \partial_{k}^{*}(q) \Delta^{-1} \Lambda\left(q^{-1}\right)-\Lambda\left(q \partial_{k}^{*}\left(q^{-1}\right)\right) \\
& =-\Lambda(q)\left(\Lambda\left(q^{-1} \partial_{k}^{*}(q)\right) \Delta+\Delta\left(q^{-1} \partial_{k}^{*}(q)\right)\right) \Delta^{-1} \Lambda\left(q^{-1}\right)-\Lambda\left(q \partial_{k}^{*}\left(q^{-1}\right)\right) \\
& =-\Lambda(q) \Delta\left(q^{-1} \partial_{k}^{*}(q)\right) \Delta^{-1} \Lambda\left(q^{-1}\right) \\
& =-\Lambda(q) \Delta\left(q^{-1}\left(\left(M_{\Delta} L^{k}\right)_{+}+\frac{k}{2} L^{k-1}+X_{k-1}^{(1)}\right)\right)(q) \Delta^{-1} \Lambda\left(q^{-1}\right) .
\end{aligned}
$$


Theorem 5.2. The additional symmetry flows (4.2) for the cdKP hierarchy $((2.14)$ for $m=1, l=1)$ commute with the difference type gauge transformation $T_{D}(q)$ preserving the form of $c d K P$, up to shifting of (4.2) by ordinary time flows, that is,

$$
\partial_{k}^{*} \widetilde{L}=\left[-\left(\widetilde{M}_{\Delta} \widetilde{L}^{k}\right)_{-}+\widetilde{X}_{k-1}^{(1)}, \widetilde{L}\right]+\frac{\partial \widetilde{L}}{\partial t_{k-1}} .
$$

Proof. Firstly, by (4.2),

$$
\begin{aligned}
\partial_{k}^{*} \widetilde{L} & =\partial_{k}^{*}\left(T_{D}(q) L T_{D}^{-1}(q)\right) \\
& =\partial_{k}^{*} T_{D}(q) \cdot L T_{D}(q)^{-1}+T_{D}(q) \partial_{k}^{*} L \cdot T_{D}^{-1}(q)-T_{D}(q) L T_{D}^{-1}(q) \cdot \partial_{k}^{*} T_{D}(q) \cdot T_{D}^{-1}(q) \\
& =\partial_{k}^{*} T_{D}(q) \cdot T_{D}^{-1}(q) \widetilde{L}+T_{D}(q)\left[-\left(M_{\Delta} L^{k}\right)_{-}+X_{k-1}^{(1)}, L\right] T_{D}^{-1}(q)-\widetilde{L} T_{D}^{-1}(q) \cdot \partial_{k}^{*} T_{D}(q) \\
& =\left[T_{D}(q)\left(-\left(M_{\Delta} L^{k}\right)_{-}+X_{k-1}^{(1)}\right) T_{D}^{-1}(q)+\partial_{k}^{*} T_{D}(q) \cdot T_{D}(q)^{-1}, \widetilde{L}\right] .
\end{aligned}
$$

Then with the help of (5.8), (5.9), and the following useful formula (3.9) in [27], we have

$$
\begin{aligned}
& T_{D}(q)\left(-\left(M_{\Delta} L^{k}\right)_{-}+X_{k-1}^{(1)}\right) T_{D}^{-1}(q)+\partial_{k}^{*} T_{D}(q) \cdot T_{D}^{-1}(q) \\
= & -T_{D}(q)\left(M_{\Delta} L^{k}\right)_{-} T_{D}^{-1}(q)+T_{D}(q) X_{k-1}^{(1)} T_{D}^{-1}(q)+\partial_{k}^{*} T_{D}(q) \cdot T_{D}^{-1}(q) \\
= & -T_{D}(q)\left(M_{\Delta} L^{k}\right)_{-} T_{D}^{-1}(q)+\widetilde{X}_{k-1}^{(1)}+\left(T_{D}(q)\left(X_{k-1}^{(1)}+\frac{k}{2} L^{k-1}\left(q_{i}\right)\right)\right)(q) \Delta^{-1} \Lambda\left(q^{-1}\right) \\
& -\left(\widetilde{L}^{k-1}\right)_{-}-T_{D}(q)\left\{\left(M_{\Delta} L^{k}\right)_{+}+\frac{k}{2} L^{k-1}+X_{k-1}^{(1)}\right\}\left(T_{D}^{-1}(q)\right) \\
= & -\left(\widetilde{M}_{\Delta} \widetilde{L}^{k}\right)_{-}+\widetilde{X}_{k-1}^{(1)}-\left(\widetilde{L}^{k-1}\right)_{-},
\end{aligned}
$$

where the following relation [28] is used,

$$
\left(\widetilde{L}^{k-1}\right)_{-}=\sum_{j=0}^{k-2} \widetilde{L}^{k-j-2}(\widetilde{q}) \Delta^{-1} \widetilde{L}^{j}(\widetilde{r}) .
$$

In the above process,

$$
\begin{aligned}
& -T_{D}(q)\left(M_{\Delta} L^{k}\right)_{-} T_{D}^{-1}(q)-T_{D}(q)\left(M_{\Delta} L^{k}\right)_{+}\left(T_{D}^{-1}(q)\right) \\
= & -T_{D}(q)\left(M_{\Delta} L^{k}\right) T_{D}^{-1}(q)+T_{D}(q)\left(M_{\Delta} L^{k}\right)_{+} T_{D}^{-1}(q)-T_{D}(q)\left(M_{\Delta} L^{k}\right)_{+}\left(T_{D}^{-1}(q)\right) \\
= & -\left(\widetilde{M}_{\Delta} \widetilde{L}^{k}\right)+\left(\widetilde{M}_{\Delta} \widetilde{L}^{k}\right)_{+} \\
= & -\left(\widetilde{M}_{\Delta} \widetilde{L}^{k}\right)_{-}
\end{aligned}
$$

which can be got by means of the identities (3.28) of Lemma.3.3.

At last, the substituting (5.12) into (5.11) gives rise to

$$
\begin{aligned}
\partial_{k}^{*} \widetilde{L} & =\left[-\left(\widetilde{M_{\Delta}} \widetilde{L}^{k}\right)_{-}+\widetilde{X}_{k-1}^{(1)}-\left(\widetilde{L}^{k-1}\right)_{-}, \widetilde{L}\right] \\
& =\left[-\left(\widetilde{M_{\Delta}} \widetilde{L}^{k}\right)_{-}+\widetilde{X}_{k-1}^{(1)}, \widetilde{L}\right]-\left[\left(\widetilde{L}^{k-1}\right)_{-}, \widetilde{L}\right] \\
& =\left[-\left(\widetilde{M_{\Delta}} \widetilde{L}^{k}\right)_{-}+\widetilde{X}_{k-1}^{(1)}, \widetilde{L}\right]+\frac{\partial \widetilde{L}}{\partial t_{k-1}} .
\end{aligned}
$$


Remark 2: When $m$ is not 1, Theorem 5.1 and Theorem 5.2 do not hold. We make a example for the integral type transformations $T_{I}(r)$ in Theorem 5.1. In fact, when $m>1$, (5.2) will become into

$$
\begin{aligned}
T_{I}\left(r_{a}\right) X_{k-1}^{(1)} T_{I}\left(r_{a}\right)^{-1}= & \widetilde{X}_{k-1}^{(1)}+\sum_{j=0}^{k-2} \widetilde{L}^{k-j-2}\left(\widetilde{q}_{a}\right) \Delta^{-1} \widetilde{L}^{j}\left(\widetilde{r}_{a}\right) \\
& +\Lambda^{-1}\left(r_{a}^{-1}\right) \Delta^{-1}\left\{T_{I}\left(r_{a}\right)^{*-1}\left(X_{k-1}^{(1)}-\frac{k}{2} L^{k-1}\right)^{*}\left(r_{a}\right)\right\},
\end{aligned}
$$

where $r_{a}$ is one of the adjoint eigenfunctions in (2.14). The term $\sum_{j=0}^{k-2} \widetilde{L}^{k-j-2}\left(\widetilde{q_{a}}\right) \Delta^{-1} \widetilde{L}^{j}\left(\widetilde{r_{a}}\right)$ in (5.14) will cause the difficulty for the proof of Theorem 5.1. Actually, for $m>1$ [13],

$$
\left(\widetilde{L}^{k-1}\right)_{-}=\sum_{a=1}^{m} \sum_{j=0}^{k-2} \widetilde{L}^{k-j-2}\left(\widetilde{q_{a}}\right) \Delta^{-1} \widetilde{L}^{j}\left(\widetilde{r_{a}}\right),
$$

so the term $\sum_{j=0}^{k-2} \widetilde{L}^{k-j-2}\left(\widetilde{q_{a}}\right) \Delta^{-1} \widetilde{L}^{j}\left(\widetilde{r_{a}}\right)$ can not be written as $\left(\widetilde{L}^{k-1}\right)_{-}$like the condition of $m=1$, which is corresponding to

$$
\partial_{t_{k-1}} \widetilde{L}=-\left[\widetilde{L}_{-}^{k-1}, \widetilde{L}\right]
$$

So for $m>1$, Theorem 5.1 does not hold.

Remark 3: For simplicity, it is proved for $l=1$ in Theorem 5.1 and Theorem 5.2. But for $l \neq 1$ it is also satisfied for Theorem 5.1 and Theorem 5.2.

\section{Conclusions And Discussions}

After some technical identities of two types gauge transformations of the cdKP hierarchy, the interplay of the integral type gauge transformation $T_{I}$ and the difference type gauge transformation $T_{D}$ with the additional symmetry at the instance of the cdKP hierarchy are obtained in Theorem 5.1, Theorem 5.2 (see $(5.4,5.10)$ ), which preserves the form of the additional symmetry of the cdKP hierarchy, up to shifting of the corresponding additional flows by ordinary time flows. Nonetheless the shifting is different from the integral type gauge transformation and the difference type gauge transformation of the cdKP hierarchy. It reflects one of the intrinsic features for the cdKP hierarchy. These results provide a mathematical background from the point of view of integrable systems of the potential applications in physics for the additional symmetry flows of the cdKP hierarchy.

\section{Acknowledgments}

This work is supported by the National Natural Science Foundation of China under Grant Nos.11271210 and 11301526, K. C. Wong Magna Fund in Ningbo University, Natural Science Foundation of Ningbo under Grant No. 2014A610029 and Science Fund of Ningbo University (Nos. XYL14028 and XKL14D2043). One of the authors (MH) is supported by Erasmus Mundus Action 2 EXPERTS III and would like to thank Prof. Antoine Van Proeyen for many helps. The authors thanks the valuable suggestion of the Referees.

\section{References}

[1] M. Adler, T. Shiota and P. van Moerbeke, A Lax representation for the vertex operator and the central extension, Comm. Math. Phys. 171 (1995), 547-588. 
[2] H. Aratyn, E. Nissimov and S. Pacheva, Virasoro symmetry of constrained KP hierarchies, Phys. Lett. A 228 (1997), 164-175 (arXiv:hep-th/9602068).

[3] L. L. Chau, J. C. Shaw and H. C. Yen, Solving the KP hierarchy by gauge transformations, Comm. Math. Phys. 149 (1992), 263-278.

[4] L. L. Chau, J. C. Shaw and M. H. Tu, Solving the constrained KP hierarchy by gauge transformations, J. Math. Phys. 38 (1997), 4128-4137.

[5] H. H. Chen, Y. C. Lee and J. E. Lin, On a new hierarchy of symmetry for the Kadomtsev-Petviashvili equation, Physica D 9 (1983), 439-445.

[6] Y. Cheng, Constraints of the KP hierarchy, J. Math. Phys. 33 (1992), 3774-3782.

[7] Y. Cheng and Y. S. Li, The constraint of the KP equation and its special solutions, Phys. Lett. A 157 (1991), 22-26.

[8] J. P. Cheng, K. L. Tian and J. S. He, The additional symmetries for the BTL and CTL hierarchies, J. Math. Phys. 52 (2011), 053515.

[9] J. P. Cheng, M. H. Li and J. S. He, The Virasoro action on the tau function for the constrained discrete KP hierarchy, J. Nonlinear Math. Phys. 20 (2013), 529-538.

[10] E. Date, M. Jimbo, M. Kashiwara and T. Miwa. Transformation groups for soliton equations, in Nonlinear integrable systems - classical theory and quantum theory, edited by M. Jimbo and T. Miwa (World Scientific, Singapore, 1983), 39-119.

[11] L. A. Dickey, Soliton equations and Hamiltonian systems (2nd Edition) (World scientific, Singapore, 2003).

[12] L. A. Dickey, On additional symmetries of the KP hierarchy and Sato's Bäcklund transformation, Comm. Math. Phys. 167 (1995), 227-233.

[13] B. Enriquez, A. Yu. Orlov and V. N. Rubtsov, Dispersionful analogues of Benney's equations and $N$ wave systems, Inverse Problems 12 (1996), 241-250 (arXiv:solv-int/9510002).

[14] A. S. Fokas and B. Fuchssteiner, The hierarchy of the Benjamin-Ono equation, Physics Letters A 86 (1981), 341-345.

[15] W. Fu, L. Huang, K. M. Tamizhmani and D. J. Zhang, Integrable properties of the differential-difference Kadomtsev-Petviashvili hierarchy and continuum limits, Nonlinearlity 26 (2013), 3197-3229.

[16] B. Fuchssteiner, Mastersymmetries, higher order time dependent symmetries and conserved densities of nonlinear evolution equations, Prog. Theor. Phys. 70 (1983), 1508-1522.

[17] L. Haine and P. Iliev, Commutative rings of difference operators and an adelic flag manifold, Int. Math. Res. Not. 6 (2000), 281-323.

[18] J. S. He, Y. H. Li and Y. Cheng, $q$-deformed KP hierarchy and $q$-deformed constrained KP hierarchy, SIGMA 2 (2006), 060.

[19] J. S. He, Y. S. Li and Y. Cheng, The determinant representation of the gauge transformation operators, Chin. Ann. Math.B 23 (2002), 475-486.

[20] J. S. He, Y. S. Li and Y. Cheng. Two choices of the gauge transformation for the AKNS hierarchy through the constrained KP hierarchy, J. Math. Phys. 44 (2003), 3928 -3960.

[21] J. S. He, Z. W. Wu and Y. Cheng, Gauge transformations for the constrained CKP and BKP hierarchies, J. Math. Phys. 48 (2007), 113519.

[22] J. S. He, K. L. Tian, A. Foerster and W. X. Ma, Additional symmetries and string equation of the CKP hierarchy, Lett. Math. Phys. 81 (2007), 119-134.

[23] S. Kanaga Vel, K.M. Tamizhmani, Lax pairs, symmetries and conservation laws of a differentialdifference equation-Sato's approach, Chaos, Solitons Fractals 8 (1997), 917-31.

[24] B. Konopelchenko, J. Sidorenko and W. Strampp, (1+1)-dimensional integrable systems as symmetry constraints of (2+1)-dimensional systems, Phys. Lett. A 157 (1991), 17-21.

[25] B. A. Kupershimidt, Discrete Lax equations and differential-difference calculus, Astérisque 123 (1985), $1-212$.

[26] C. Z. Li, J. S. He and Y. C. Su, Block type symmetry of bigraded Toda hierarchy, J. Math. Phys. 53 (2012), 013517.

[27] M. H. Li, J. P. Cheng and J. S. He, The gauge transformation of the constrained semi-discrete KP hierarchy, Mod. Phys. Lett. B 27 (2013), 1350043. 
[28] M. H. Li, C. Z. Li, K. L. Tian, J. S. He, Y. Cheng, Virasoro type algebraic structure hidden in the constrained discrete Kadomtsev-Petviashvili hierarchy, J. Math. Phys. 54 (2013), 043512.

[29] S. W. Liu and Y. Cheng, Sato Backlund transformation, additional symmtries and ASvM formular for the discrete KP hierarchy, J. Phys. A: Math. Theor. 43 (2010), 135202.

[30] S. W. Liu, Y. Cheng and J. S. He, The determinant representation of the gauge transformation for the discrete KP hierarchy, Sci. China Math. 53 (2010), 1195-1206.

[31] I. Loris and R. Willox, On solutions constrained KP equations, J. Math. Phys. 38 (1997), 283-291.

[32] P. van Moerbeke, Integrable fundations of string theory, in Lectures on Integrable systems, edited by $\mathrm{O}$. Babelon, P. Cartier, Y. Kosmann-Schwarzbach (World Scientific, Singapore, 1994), 163-267.

[33] J. J. C. Nimmo, Darboux transformations from reductions of the KP hierarchy, in Nonlinear Evolution Equations and Dynamical Systems, edited by V. G. Makhankov, A. R. Bishop, and D. D. Holm (World Scientific, Singapore, 1995), 168-177.

[34] W. Oevel, Darboux theorems and Wronskian formulas for integrable system I: constrained KP flows, Physica A 195 (1993), 533-576.

[35] W. Oevel, Darboux transformations for integrable lattice systems, in Nonlinear Physics: Theory and Experiment, edited by E.Alfinito, L. Martina and F.Pempinelli (World Scientific, Singapore, 1996), 233-240.

[36] W. Oevel and B. Fuchssteiner, Explicit formulas for symmetries and conservation laws of the Kadomtsev-Petriashvili equations, Phys. Lett.A 88 (1982), 323-327.

[37] W. Oevel and C. Rogers, Gauge transformations and reciprocal links in $2+1$ dimensions, Rev. Math. Phys. 5 (1993), 299-330.

[38] A. Yu. Orlov and E. I. Schulman, Additional symmetries for integrable systems and conformal algebra repesentation, Lett. Math. Phys. 12 (1986), 171-179.

[39] H. F. Shen and M. H. Tu, On the constrained B-type Kadomtsev-Petviashvili hierarchy: Hirota bilinear equations and Virasoro symmetry, J. Math. Phys. 52 (2011), 032704.

[40] K. L. Tian, J. S. He, J. P. Cheng and Y. Cheng, Additional symmetries of constrained CKP and BKP hierarchies, Sci. China Math. 54 (2011), 257-268.

[41] M. H. Tu, On the BKP hierarchy: Additional symmetries, Fay identity and Adler-Shiota- van Moerbeke formula, Lett. Math. Phys. 81 (2007), 91-105.

[42] M. H. Tu, J. C. Shaw, and C. R. Lee, On Darboux - Backlund transformations for the $q$-deformed Korteweg - de Vries hierarchy, Lett. Math. Phys. 49 (1999), 33 - 45 (arXiv:solv-int/9811004).

[43] R. Willox and I. Loris, KP constraints from reduced multi-component hierarchies, J. Math. Phys. 40 (1999), 6501-6525.

[44] R. Willox, I. Loris and C. R. Gilson, Binary Darboux transformations for constrained KP hierarchies, Inverse Problems 13 (1997), 849-865.

[45] Y. J. Zhang and Y. Cheng, Solutions for the Vector k-constrained KP Hierarchy, J. Math. Phys. 35 (1994), 5869-5884. 